\title{
Evolutionary Game Based Access Class Barring for Machine-to-Machine Communications
}

\author{
Tingsong Jiang, Xu Tan, Xi Luan, Xiaoning Zhang, Jianjun Wu \\ Institution of Advanced Communications, Peking University, Beijing, China \\ tingsong@pku.edu.cn,just@pku.edu.cn
}

\begin{abstract}
Machine-to-machine (M2M) communication faces the most critical challenge that when a massive number of MTC devices perform random access, there will be severe congestions. 3GPP developed a network coordinated random access stabilization scheme known as the access class barring(ACB) to deal with the problem in LTE-Advanced. However, MTC devices in overlapped areas still suffer severe access delays due to the noncooperation and individual setting of $\mathrm{ACB}$ factor among BSs. We propose evolutionary game based ACB algorithm to help MTC devices alleviate congestions and thus eliminate substantial defects in the ordinary ACB. Finally, the performance of the evolutionary game based ACB algorithm is analysed, and simulation results show the convergence and effectiveness of the proposed algorithm.
\end{abstract}

Keywords - Evolutionary Game, Access Class Barring (ACB), M2M communications, Radom Access

\section{INTRODUCTION}

Machine-to-machine communication has been one of the focuses recent years since the demand of machine-type communication is increasing rapidly [1]-[3]. 3GPP is active in developing M2M-related standards for LTE networks.

In 3GPP and IEEE 802.16p, it is proposed that each MTC device attaches to the existing cellular infrastructures, which provide higher layers connections among MTC devices. M2M communications should support a massive number of MTC devices [2]-[4]. However, the most critical and open challenge lies in the air interface by which random access are performed to prevent access congestions of the cellular infrastructure. In LTE-Advanced, to alleviate congestions, a network coordinated random access stabilization scheme known as the access class barring (ACB) is proposed [5], [6].

In the $\mathrm{ACB}$, when the random access procedure is initialized, the BS broadcasts an ACB factor $\mathrm{p}$ between 0 and 1 to all MTC devices. Each active MTC device generates a random number between 0 and 1 . If this number is less than the ACB factor, then the active MTC device proceeds to the random access procedure; otherwise, it is barred for a barring time duration and wait for the next available turn. Therefore, the BS can control the congestion by controlling the ACB factor $\mathrm{p}$.

However, M2M communication has problems in the ordinary ACB because each BS individually determines the $\mathrm{ACB}$ factor $\mathrm{p}$ for the cell formed by the BS itself. If a severe congestion occurs in a cell, the BS may set its $p$ to an extremely low value, which means there are a lot of MTC devices barred and thus the access delay is undoubtedly unacceptable. Cooperative ACB scheme is proposed to optimize the ACB factor using iterations [7]. In the ordinary $\mathrm{ACB}$, delay is by no means to be improved.

When analysing the competition behaviors of users in the cell, game theory can be useful and efficient tools. Among different kinds of games, the evolutionary game is suitable for problems with multiple Nash equilibriums [8], [9]. In this paper, we propose an evolutionary game based ACB scheme where evolutionary game is brought in to analyse the behaviors of the MTC devices and all ACB parameters of all BSs are jointly optimized based on levels of congestions in each BS to substantially enhance the performance of the ordinary ACB. Our simulation results show that the evolutionary game based ACB scheme can alleviate congestions efficiently, and the delay is significantly improved.

\section{SySTEM MODEL}

We consider the random access of M2M communications in LTE-Advanced with M BSs and $\mathrm{N}$ active MTC devices. We propose a framework that all MTC devices are able to access the BS unattached by the MTC device when the MTC device locates within the overlapped coverage area of multiple BSs (A1,A2 and A3) while the MTC devices are limited to access the attached BS in ordinary ACB. The BS has a large coverage area and radiation power that can provide wireless service to a lot of users.

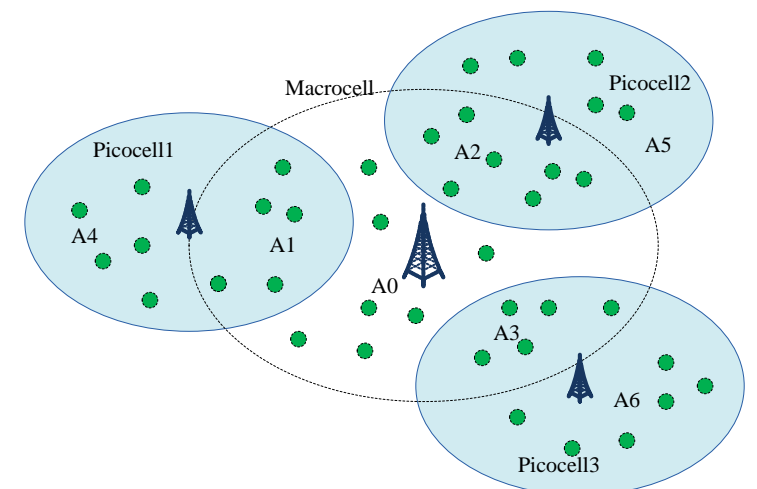

Figure 1. System model of MTC devices in LTE-Advanced netwok 
In the ordinary $\mathrm{ACB}$, each $\mathrm{BS}$ sets its $\mathrm{ACB}$ factor $p_{m}=1 / N_{m}$ (where $p_{m}$ is the ACB factor of the mth $\mathrm{BS}$ ) individually in order to maximize its throughput. However, the delay experienced by MTC devices attached to the $m t h$ BS may be unacceptable when $p_{m}$ requires to be set to an extremely small value under a large $N_{m}$.

Such scenario happens when a lot of MTC devices are located in the overlapped areas of multiple BSs, MTC devices should select one BS to perform random access. Our aim is to guide the MTC devices to select the proper BS reasonably and rationally and decentralize the MTC devices in case of congestion. Evolutionary game is a useful method for cell selection and when the evolution reaches equilibrium, every BS has a proper crowd of MTC devices to perform random access. At this time, each BS sets its ACB factor according to the numbers waiting to perform random access, therefore it can alleviate congestion.

\section{ACB BASED ON EVOLUTIONARY GAME}

In this section, we propose an evolutionary game based ACB scheme. In the evolutionary game, MTC devices who can detect and select the available base stations to perform random access are the players of the game. The population is a set of users in the game. Strategies are the users' choices of different base stations and payoff is the utility obtained by each user. Replicator dynamics and evolutionary equilibrium are two main concepts of the evolutionary game. Let $\mathrm{N}$ be the population size of the game and $n_{i}$ be the number of players select strategy $i$. We use $\pi_{i}(t)$ to denote the utility of players select strategy $i$ and $\bar{\pi}(t)$ to denote the average utility of the entire population. The proportion of players selecting strategy $i$ is $x_{i}=n_{i} / N$. The replicator dynamics and evolutionary equilibrium can be defined as follows:

- Replicator dynamics: The replicator dynamics model the rate of strategy change, which can be expressed as follows:

$$
\dot{x}_{i}(t)=x_{i}(t)\left(\pi_{i}(t)-\bar{\pi}(t)\right)
$$

- Evolutionary equilibrium: The evolutionary equilibrium is defined as the set of stable points of the replicator dynamics, where the utility of all users are identical and no player will change its current strategy because the rate of strategy change is zero $\left(\dot{x}_{i}=0, \forall i\right)$.

We use $N^{a}$ to denote the number of users in area $a$ and $x_{i}^{a}$ to denote the proportion of users in area $a$ who select base station $i$ as the service provider. The utility function of each user waiting to connect base station $i$ can be expressed as follows:

$$
\pi_{i}(\mathbf{x})=U\left(\frac{C_{i}}{\sum_{a \in \Omega_{i}} N^{a} x_{i}^{a}}\right)-P\left(\sum_{a \in \Omega_{i}} N^{a} x_{i}^{a}\right)
$$

where $\mathbf{x}$ denotes the vector of proportion of users choosing different networks in all areas.

$$
\begin{gathered}
U\left(\frac{C_{i}}{\sum_{a \in \Omega_{i}} N^{a} x_{i}^{a}}\right)=\log \left(1+\frac{C_{i}}{\sum_{a \in \Omega_{i}} N^{a} x_{i}^{a}}\right) \\
P\left(\sum_{a \in \Omega_{i}} N^{a} x_{i}^{a}\right)=p_{i} \cdot \sum_{a \in \Omega_{i}} N^{a} x_{i}^{a}
\end{gathered}
$$

where $C_{i}$ is the channel capacity provided by the base station $i$ which is allocated to the users and $\Omega_{i}$ is the coverage area of base station $i$. We assume that all users in the same coverage area who select the same base station are allocated with the equal channel capacity, so for each user (3) can be seen as the revenue of each user gained from the base station and (4) is the payoff for the service.

\section{A. Utility Function}

In Fig. 1, the coverage areas of macrocell and picocell overlap in some areas. From (2), (3) and (4), we know that users in the coverage area of one base station will share its total channel capacity equally. The utility functions are expressed as follows:

Macrocell:

$$
\pi_{M B}=U\left(\frac{C_{M B}}{n_{M B}}\right)-P\left(n_{M B}\right)
$$

where $n_{M B}=N^{0} x_{M B}^{0}+N^{1} x_{M B}^{1}+N^{2} x_{M B}^{2}+N^{3} x_{M B}^{3}$,

Picocell1 Picocell3:

$$
\pi_{P 1}=U\left(\frac{C_{P 1}}{n_{P 1}}\right)-P\left(n_{P 1}\right)
$$

where $n_{P 1}=N^{1} x_{P 1}^{1}+N^{4} x_{P 1}^{4}$,

$$
\pi_{P 2}=U\left(\frac{C_{P 2}}{n_{P 2}}\right)-P\left(n_{P 2}\right)
$$

where $n_{P 2}=N^{2} x_{P 2}^{2}+N^{5} x_{P 2}^{5}$,

$$
\pi_{P 3}=U\left(\frac{C_{P 3}}{n_{P 3}}\right)-P\left(n_{P 3}\right)
$$

where $n_{P 3}=N^{3} x_{P 3}^{3}+N^{6} x_{P 3}^{6}$.

\section{B. Replicator Dynamics}

MTC devices in overlapped areas are divided into different groups and replicator dynamics can show the change of numbers in each group over time. The group of users in area $a$ can choose among the available access networks (i.e., select strategy $i, i=M B, P 1 \sim P 3)$. We assume that the utilities of every user chosen the same BS are the same regardless of their areas, that is

$$
\pi_{i}^{a}=\pi_{i}, \forall a=1,2,3
$$

Replicator dynamics can be denoted as 


$$
\dot{x}_{i}^{a}=\lambda x_{i}^{a}\left(\pi_{i}^{a}-\bar{\pi}^{a}\right)
$$

where $\lambda$ is the multiplier of the difference of user's utility and the average utility. Users in A1 can select Macrocell and Picocell1, the replicator dynamics of A1 can be denoted as

$$
\begin{gathered}
\dot{x}_{M B}^{1}=\lambda x_{M B}^{1}\left(\pi_{M B}^{1}-\bar{\pi}^{1}\right) \\
=\lambda x_{M B}^{1}\left(1-x_{M B}^{1}\right)\left(\pi_{M B}^{1}-\pi_{P 1}^{1}\right) \\
\dot{x}_{P 1}^{1}=\lambda x_{P 1}^{1}\left(1-x_{P 1}^{1}\right)\left(\pi_{P 1}^{1}-\pi_{M B}^{1}\right) \\
x_{M B}^{1}+x_{P 1}^{1}=1
\end{gathered}
$$

We can obtain replicator dynamics in A2 and A3 similarly

$$
\begin{aligned}
& \dot{x}_{M B}^{2}=\lambda x_{M B}^{2}\left(1-x_{M B}^{2}\right)\left(\pi_{M B}^{2}-\pi_{P 2}^{2}\right) \\
& \dot{x}_{P 2}^{2}=\lambda x_{P 2}^{2}\left(1-x_{P 2}^{2}\right)\left(\pi_{P 2}^{2}-\pi_{M B}^{2}\right) \\
& \dot{x}_{M B}^{3}=\lambda x_{M B}^{3}\left(1-x_{M B}^{3}\right)\left(\pi_{M B}^{3}-\pi_{P 3}^{3}\right) \\
& \dot{x}_{P 3}^{3}=\lambda x_{P 3}^{3}\left(1-x_{P 3}^{3}\right)\left(\pi_{P 3}^{3}-\pi_{M B}^{3}\right)
\end{aligned}
$$

\section{Evolutionary Equilibrium}

Evolutionary equilibrium is considered as the solution of the game, which is a fixed point of replicator dynamics. When the equilibrium point is reached, payoffs of all users in area $a$ are identical and the proportions of all users in each group will be unchanged anymore. The utility in each group will also be identical and users will not try to change their choice since no higher utility will be obtained. Evolutionary equilibrium is the key reference for ACB.

\section{Evolutionary Gamed based ACB}

The evolutionary gamed based ACB algorithm is described in Table 1. MTC devices in A1 are going to perform random access, and their choice is either Macrocell or Picocell1. First, MTC devices in A1 play the evolutionary game until the equilibrium is achieved and thus the proportion of each BS's candidates waiting to perform random access is obtained. MTC devices are divided into different "buffer zones" of each BS. Then each BS sets its ACB factor according to its buffer zone size. Next, each MTC device waiting to access the mth $\mathrm{BS}$ randomly draws a number $\mathrm{q} \in[0,1]$. If $\mathrm{q}<\mathrm{p}$, then the active MTC device proceeds to the random access procedure; otherwise, the active MTC device is barred for a barring time duration and waits for the next turn. When at least two active MTC devices are performing random access, they are also barred for a barring time in order to guarantee one user one turn. If the active MTC device accesses the BS successfully, then the BS decreases its $\mathrm{ACB}$ factor to ensure the proper probability.

\section{IV.SIMULATION RESULTS}

We consider a network containing one Macrocell and three Picocells. To evaluate the performance of the proposed evolutionary game based ACB, we consider simulation parameters for LTE MTC devices [10] that had been adopted by 3GPP. Conventionally, picocells are deployed at the hot spot to share traffic load of the Macrocell, so three picocells are deployed at the boundary of the central Macrocell with wrap-around. In this simulation, the coverage of three picocells is deployed by $20 \%$ among total N MTC devices separately; the central Macrocell has a proportion of $40 \%$. The period of RACH is $5 \mathrm{~ms}$. We assume that $p_{i}$ in (4) is 0.08 for Macrocell and 0.1 for each Picocell., the multiplier of replicator dynamics is $\lambda=1$.

When active MTC devices are trying to access the BS, the performance of the evolutionary game is important. Given the initial proportions of users in area $a$ as $x_{M B}^{a}=0.5, x_{P a}^{a}=0.5$, the changes of proportion are plotted respectively in Fig.2. They can select their service providers according to the utilities that the users obtain. Please note that in this step MTC devices only show their intensions to access each BS rather than a real connection. The dynamics of utilities in each group is shown in Fig.3. We observe that all users reach the same utilities at the evolutionary equilibrium, therefore no users will change its current service provider since no higher utility will be rewarded. Consequently the evolutionary equilibrium is stable and convergent.

The average delay and worst delay in this situation is shown in Fig.4. The average delay is the delay performance averaged over all N MTC devices, while the worst case delay is the largest delay among N MTC devices. We can observe that the proposed evolutionary game based ACB has improvement compared with ordinary $\mathrm{ACB}$ both in the average delay and worst delay due to the game procedure.

TABLE 1. Evolutionary Game BASEd ACB Schemes

Evolutionary Game Based ACB Scheme
1: Initialize the proportion vector waiting to choose the
mth BS to access: $\mathbf{x}_{B S_{m}}^{a}$.
2: MTC devices play evolutionary game according to the
utility function until the equilibrium is achieved.
3: The $m t h$ BS sets ACB factor according to the
equilibrium $x_{B S_{m}}^{a}$. The $m t h$ BS broadcasts the ACB
factor $p_{m}$ to inform active MTC devices to access.
4: While $N_{a}>0$ do
5: Each active MTC device draws a random number $q_{n}$.
6: if $q_{n}<p_{m}$,
7: then it performs random access.
8: else wait for next turn;
9: end if
10: if more than one MTC device access the same BS
11: then they are barred and wait for next turn.
12: else perform random access, $N_{a}=N_{a}-1$.
13: Update ACB factor $p_{m}=1 / \sum_{m} N_{a} \cdot x_{B S m}^{a}$
14: end if
15: end while




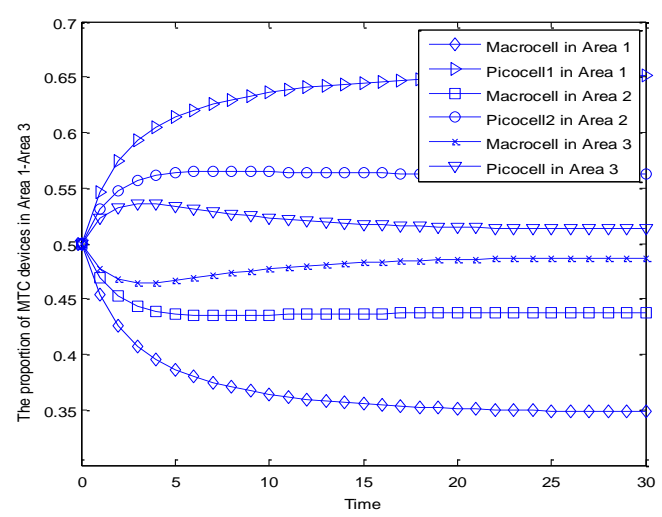

Figure 2. Evolutionary process of MTC devices toward evolutionary equilibrium.

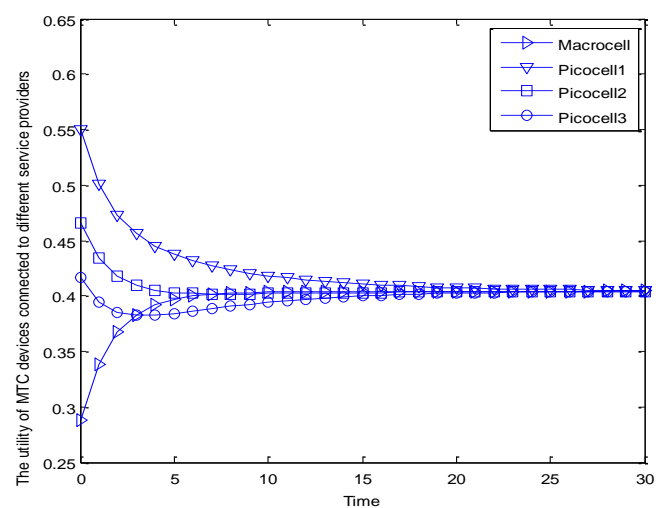

Figure 3. Dynamics of utilities in the evolutionary game.

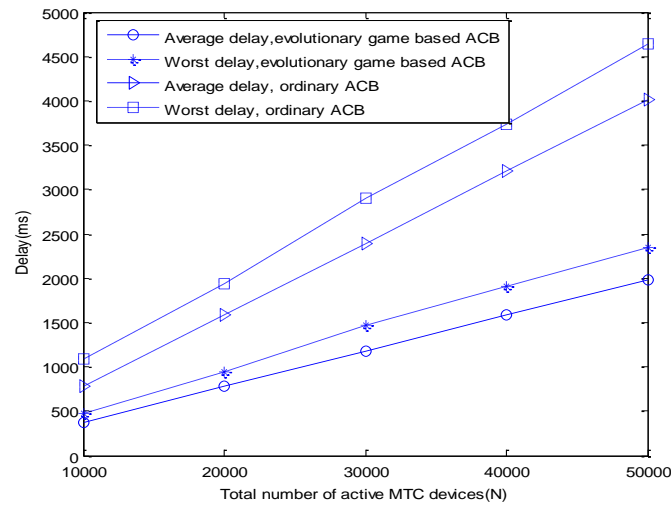

Figure 4. The average delay and worst delay in proposed evolutionary game based ACB and ordinary ACB

\section{Conclusions}

In this paper, we propose an evolutionary game based ACB which can improve the performance of M2M communication. How to guide the MTC devices to attach proper BS and perform random access efficiently is our aim. Evolutionary equilibrium is defined as the reference to adjust ACB factor. Evolutionary game based ACB has an advantage that the complexity is low, besides it has no impacts on the system architecture of LTE-Advanced, thus we can apply it to LTEAdvanced. Simulation results show that our proposed algorithm has effective improvement of the access delay which is the most critical issue of M2M communication using ACB.

\section{ACKNOWLEDGMENT}

This work is partly supported by the National Science Foundation of China (Grant No. NFSC \#61071083, \#61371073) and the National High-Tech Research and Development Program of China (863 Program), No.2012AA01A506. Corresponding author: Jianjun Wu, Email: just@pku.edu.cn.

\section{REFERENCES}

[1] 3GPP TR 22.868 V8.0.0, Study on facilitating machine to machine. Available:http://www.3gpp.org/ftp/Specs/archive/22\_series/22.868/22 868-800.zip

[2] 3GPP TR 23.888 V1.2.0, System improvement for machine-type communications.Available:http://www.3gpp.org/ftp/Specs/archive/23\_ series/23.888/23888-120.zip

[3] 3GPP TS 22.368 V11.1.0, Service requirements for machine-type communications.Available:http://www.3gpp.org/ftp/Specs/archive/22 _ series/22.368/22368-b10.zip

[4] IEEE 802.16ppc-10/0011r2, "IEEE 802.16p machine to machine (M2M) system requirements document (initial working document)," Sep. 2010.

[5] CATT, "R2-100182: access control of MTC devices," in $20103 G P P$ TSG RAN WG2 Meeting 68bis.

[6] ZTE, "R2-104662: MTC simulation results with specific solutions," in 2010 3GPP TSG RAN WG2 Meeting 71.

[7] S.-Y. Lien, T.-H. Liau, C.-Y. Kao, and K.-C. Chen, "Cooperative access class barring for machine-to-machine communications," IEEE Trans. on Wireless Communications, vol. 11, no. 1, pp. $27-32$, Jan. 2012

[8] Dusit Niyato and Ekram Hossain, "Dynamics of Network Selection in Heterogeneous Wireless Networks: An Evolutionary Game Approach", IEEE Trans. on VEHICULAR TECHNOLOGY, VOL. 58, NO. 4 MAY 2009

[9] Ziqiang Feng, Lingyang Song, Zhu Han, Dusit Niyato, Xiaowu Zhao: "Cell selection in two-tier femtocell networks with open/closed access using evolutionary game". WCNC 2013: 860-865

[10] 3GPP TR 37.868 V0.7.0, Study on RAN improvements for machinetypecommunications.Available:http://www.3gpp.org/ftp/tsg\_ran/WG2 \_RL2/TSGR2\_71bis/Docs/R2-106033.zip

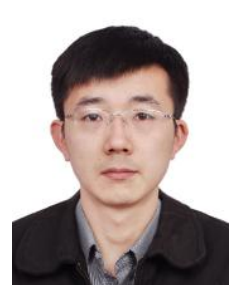

Tingsong Jiang received the bachelor degree in electronic information science and technology from Peking University, Beijing, P.R.China, in 2010. He has been an postgraduate student in Institution of Advanced Communications, Peking University, China. His research interests are in the area of physical layer in satellite mobile communications, $\mathrm{M} 2 \mathrm{M}$ communications, energy efficiency.

Jianjun Wu received his B.S., M.S. and Ph.D. degree from Peking University, Beijing, P. R. China, in 1989, 1992 and 2006, respectively. Since 1992, he has joined the School of Electronics Engineering and Computer Science, Peking University, and has been appointed as an associate professor since 2002. His research interests are in the areas of satellite communications, wireless communications, and communications signal processing. The corresponding author.Email:just@pku.edu.cn. 